\title{
Crystal structure transformation in CeRuSn as seen via the atomic pair distribution function
}

\author{
K. Prokeš,${ }^{1, *}$ S. A. J. Kimber, ${ }^{2}$ J. A. Mydosh,${ }^{3}$ and R. Pöttgen ${ }^{4}$ \\ ${ }^{1}$ Helmholtz-Zentrum Berlin für Materialien und Energie, \\ Hahn-Meitner Platz 1, M-ICMM, 14109 Berlin, Germany \\ ${ }^{2}$ European Synchrotron Radiation Facility, 6 rue Jules Horowitz, 38000 Grenoble, France \\ ${ }^{3}$ Kamerlingh Onnes Laboratory, Leiden University, 2300 RA Leiden, The Netherlands \\ ${ }^{4}$ Institut für Anorganische und Analytische Chemie, \\ Westfälische-Wilhelms-Universität Münster, Corrensstrasse 30, 48149 Münster, Germany
}

(Dated: February 4, 2014)

\begin{abstract}
We report on the atomic pair distribution function determination in the ternary compound CeRuSn using high energy synchrotron x-ray diffraction. Above room temperature CeRuSn orders in a monoclinic structure that is related to the CeCoAl type of structure by a doubling of the $c$-axis parameter. Upon cooling a very broad hysteretic structural transition has been observed in CeRuSn leading to an ill-defined structure with a main component having three times larger c parameter. The pair distribution functions collected between $90 \mathrm{~K}$ and $315 \mathrm{~K}$ suggest that the local symmetry type does not change significantly during the phase transformation. However, anomalously large atomic displacement factors suggest that a wide distribution of interatomic distances exists in the sample. Limited agreement between the data at $90 \mathrm{~K}$ and the best fit suggests that the structure with three times larger c lattice parameter with respect to the CeCoAl type can only be regarded as an approximation to the real crystal structure of CeRuSn at low temperatures.
\end{abstract}

PACS numbers: 61.05.cf, 71.28.+d, 75.25.Dk

\section{INTRODUCTION}

The crystal structure of a solid is one of the most important parameters that determines its physical properties. In particular, the global and local symmetries of the structure define the form of the Hamiltonian and thus the mathematical apparatus used in its description ${ }^{1}$.

The ternary equiatomic compound CeRuSn exhibits remarkable structural and magnetic features ${ }^{2-6}$ that are still not yet understood. Based on laboratory x-ray single crystal diffraction and several x-ray and neutron powder diffraction studies, it has been shown that at room and elevated temperatures, CeRuSn adopts a superstructure of a monoclinic $\mathrm{CeCoAl}$ type ${ }^{2}$ by a doubling along the $c$ axis leading to splitting of originally single $\mathrm{Ce}, \mathrm{Ru}$ and $\mathrm{Sn}$ crystallographic positions but preserving the space group type (transition to the maximal isomorphic subgroup of index 2). This structure is referred to as the $2 \mathrm{c}$ structure.

The two inequivalently split Ce sites, $\mathrm{Ce} 1$ and $\mathrm{Ce} 2$ have distinctly different coordination leading to different valence states, namely intermediate-valence $\mathrm{Ce}^{(4-\delta)+}(\mathrm{Ce} 1)$ and trivalent $\mathrm{Ce}^{3+}(\mathrm{Ce} 2)$ states $^{2,4,7}$. The magnetic susceptibility behavior at elevated temperatures is reported to be compatible with only part of the Ce ions being in the $\mathrm{Ce}^{3+}$ state $^{3,5,7}$. It was speculated that a charge ordering and phase separation might be responsible for the structural change below room temperature ${ }^{7}$. Other explanation makes a Kondo effect ${ }^{8}$ together with strong lattice vibrations ${ }^{9}$ responsible for the structural transition.

Bulk measurements further show that below room temperature, a hysteretic crystallographic phase transformation occurs. All previous diffraction experiments show that new superstructure peaks appear upon cooling ${ }^{3,5,10}$. The $2 \mathrm{c}$ structure is replaced by new structural modifications, the dominant mode being close to a tripling of the basic CeCoAl cell (the so-called $3 \mathrm{c}$ structure). The low-temperature crystal structure can be destroyed by soft synchrotron radiation within few minutes and the $2 \mathrm{c}$ structure can be stabilized even at $10 \mathrm{~K}$. These results show that the low-temperature structure modification is rather fragile and not well defined ${ }^{10}$.

In the case of subtle modifications in the atomic positions the atomic structure may be studied using a local structural probe ${ }^{11,12}$ such as the atomic pair distribution function (PDF) analysis ${ }^{13}$ of powder x-ray or neutron diffraction data. The chosen technique is a total scattering method because it uses all structure-relevant scattering over a large reciprocal space range of the sample. The real-space structural details are revealed through the Fourier transformation ${ }^{13}$. The PDF analysis has been proven to be a very successful technique to investigate the local structure changes in many oxide materials ${ }^{13-15}$. Among other methods capable to disclose structural changes are for instance x-ray and neutron diffraction and extended X-ray absorption fine structure (EXAFS). However, one has to state clearly that preference for diffraction experiments exists only in the case that the displacements give rise to long range order. The actual resolution is given by the minimum d-spacing measured, which is in favor of diffraction experiments. However, these are quite often also intensity limited. As our previous neutron diffraction experiments ${ }^{3}$ were unable to solve the crystal structure details at low temperature unambiguously due to a large number of parameters, synchrotron studies ${ }^{10}$ could focus only on a very limited 
d-spacing range, results of x-ray single crystal studies ${ }^{5}$ were in part in conflict with other studies and EXAFS does not have the required resolution, we have decided to perform the PDF study reported here.

\section{EXPERIMENTAL}

A polycrystalline sample has been prepared by arcmelting of stoichiometric amounts of the constituent elements under purified argon atmosphere at the Institut für Anorganische und Analytische Chemie, Universität Münster as described elsewhere ${ }^{2}$. The same polycrystalline sample has been used in previous neutron powder diffraction experiments ${ }^{3}$. The sample has been ground to a fine powder under protective argon atmosphere.

Synchrotron X-ray powder diffraction data suitable for pair distribution function analysis were collected using the ID15B beamline at the European Synchrotron Radiation Facility (ESRF), Grenoble. A wavelength of 0.1422 $\AA(87 \mathrm{KeV})$ selected using a bent Si crystal in Laue transmission geometry was used and the scattered x-rays were detected by a Mar345 image plate. The energy was calibrated using a NIST $\mathrm{CeO}_{2}$ standard powder. All diffraction images were integrated using Fit2 $\mathrm{D}^{16}$. The sample was placed in a Kapton capillary and cooled using an Oxford 700-series Cryostream. The pair distribution function was calculated using in-house software (iPDF) developed by one of the authors (SAJK), which runs as a graphical user interface within Igor Pro ${ }^{17}$. Briefly, data were corrected for background, Compton scattering, and the atomic form factor. The Compton shift, detector efficiency and incoherent fluorescence were also taken into account, before Fourier transformation according to:

$$
G(r)=\frac{2}{\pi} \int_{0}^{\infty} Q[S(Q)-1] \sin (Q r) d Q
$$

Here $\mathrm{Q}[\mathrm{S}(\mathrm{Q})-1]$ represents the properly corrected and normalized intermediate structure factor and the $r$-grid used in real space had a spacing of $0.01 \AA$. Models were fitted to the PDF data using the PDFgui package ${ }^{18}$. Model pair distribution functions were calculated using:

$$
G(r)=\frac{1}{N r} \sum_{i} \sum_{j \neq i}\left[\frac{b_{i} b_{j}}{\langle b\rangle^{2}} \delta\left(r-r_{i j}\right)\right]-4 \pi r \rho_{0}
$$

The indices $i$ and $j$ run over all atoms in the sample. The scattering powers of the different atoms are $b_{i}$ and $b_{j}$, and $r$ represents the radial distance in real space. PDFgui uses the so-called small box approximation, which implies that the first summation above only runs over the atoms within one unit cell as defined by the average crystallographic structure. This approximation makes data modelling tractable out to relatively large distances in real space.

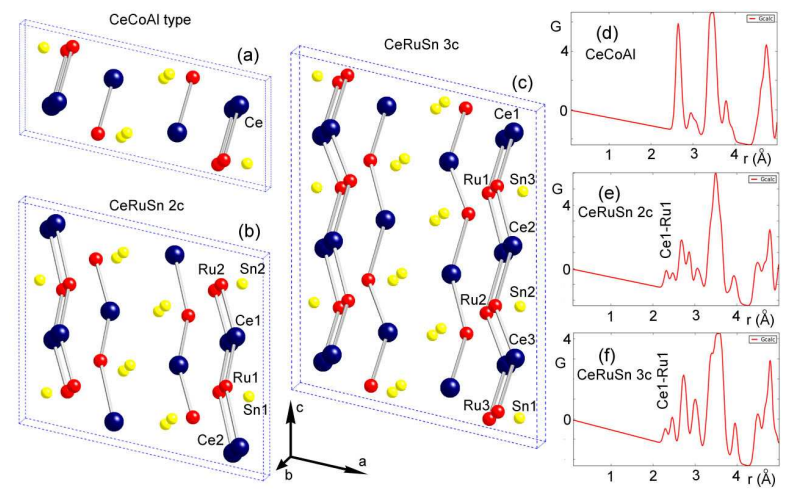

FIG. 1: (Color online) Schematic representation of the crystal structure adopted by $\mathrm{CeCoAl}$ (a) and CeRuSn at high temperatures (b) and low temperatures (c) that are doubled and trippled along the $c$-axis with respect to the CeCoAl type, respectively. All the Ce-Ru bonds smaller than $3 \AA$ are shown. In panels $(\mathrm{d}-\mathrm{f})$ we show theoretical pair distribution function of the structures shown in (a-c) assuming small isotropic displacement factors of $0.1 \AA^{2}$ for all the atoms. The shortest $\mathrm{Ce}-\mathrm{Ru}$ bonds giving the first maxima in PDFs are labeled.

\section{RESULTS AND DISCUSSION}

In Fig. 1 we show schematic representations of the crystal structure adopted by $\mathrm{CeCoAl}$ (panel a) and CeRuSn at high (panel b) and at low (panel c) temperatures as suggested in the literature together with relevant pair distribution functions, respectively ${ }^{2,3,5}$. All the structures have a monoclinic lattice with the space group $C 2 / m$. Splitting of the $4 i$ Wyckoff site positional parameters with respect to the CeCoAl type structure leads in CeRuSn to an enlargement of the c lattice parameter. Along with the doubling and trippling of the $c$-axis, the interatomic distances are modified as well. While the shortest interatomic distance within the $\mathrm{CeCoAl}$ connecting the Ce site with Co site amounts to $2.61 \AA$ (followed by another Ce-Co pair separated by marginally larger distance of $2.67 \AA$, both assuming the c-lattice parameter that is half of that found for CeRuSn at high temperatures), within the 2c structure these distances amount to $2.33 \AA$ for the Ce1-Ru1 pair and $2.48 \AA$ for the Ce1-Ru2 pair. In the case of the $3 \mathrm{c}$ structure, the shortest separation is found for the Ce1-Ru1 link that amounts to $2.29 \AA$, followed by the $\mathrm{Ce} 2-\mathrm{Ru} 2$ and $\mathrm{Ce} 2$-Ru3 pairs with $2.46 \AA$. Note that the distances for the $3 \mathrm{c}$ structure that follow from our powder neutron work ${ }^{3}$ differ somewhat from values determined by x-ray diffraction by Fikáček et al. ${ }^{5}$, most probably due to structural and lattice parameters that were determined by different methods.

To generate pair distribution function spectra for all the structures (shown in panels (d-f) of Fig. 1), we take as the input published structural parameters ${ }^{2,3,5}$ and assume an isotropic displacement factor of $0.1 \AA^{2}$, using the computer software PDFgui ${ }^{18}$. All spectra consist of clearly distinguishable peaks of different intensities (reflecting 

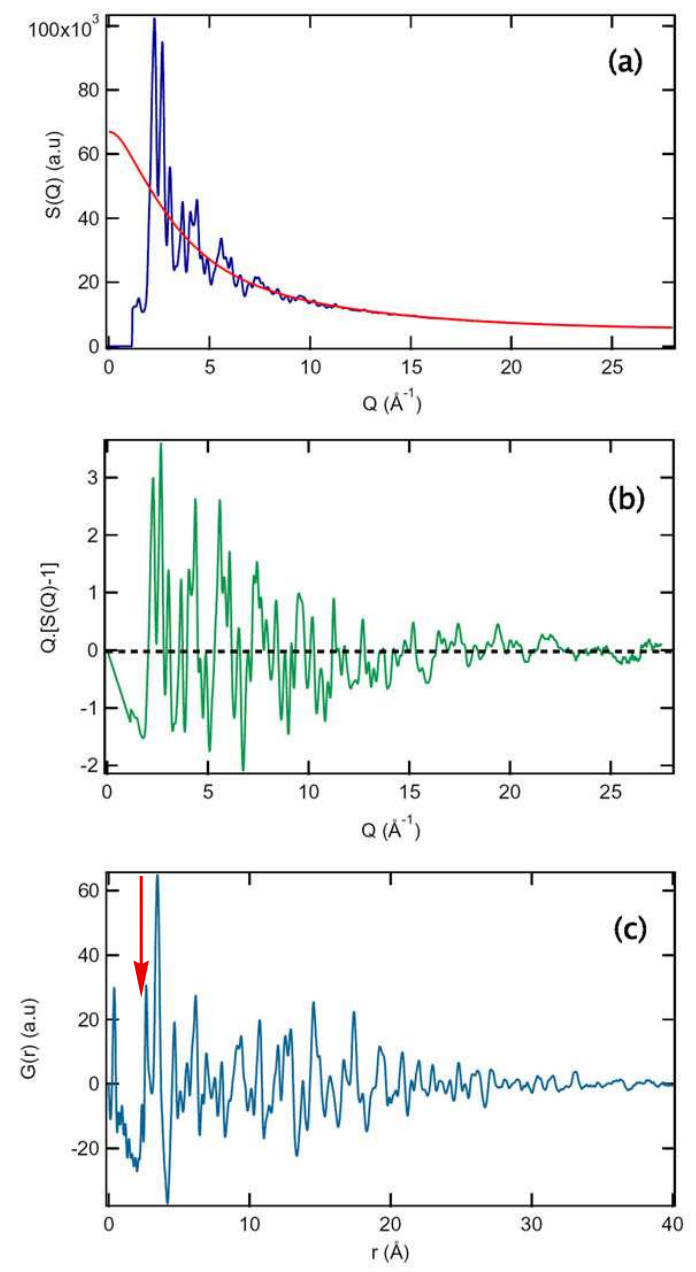

FIG. 2: (Color online) The experimental PDF data processing of CeRuSn taken at $315 \mathrm{~K}$. The raw data and the form factor are shown in (a), the intermediate structure factor in (b) and the derived pair distribution function in (c).

the different electron densities), centered at positions defined by interatomic distances. The first maxima in Fig. 1 (d-f) correspond to the interatomic distances mentioned above. Let us also note that there is no peak expected in either of the spectra significantly below $2.3 \AA$. This is even the case for the structure that has a five times larger c-lattice parameter that is reported ${ }^{5}$ to be stable on cooling between 290 and $225 \mathrm{~K}$, where the shortest distance amounts to $2.27 \AA$.

In Fig. 2 we show experimental PDF data of CeRuSn taken at $315 \mathrm{~K}$. The raw data after $2 \mathrm{D}$ integration with the corresponding form factor are shown in (a). The intermediate structure factor is shown in panel (b) and the derived atomic pair distribution function in panel (c). Several acquisition runs were combined in order to reduce the statistical fluctuations. The appearance of a maximum at $\mathrm{r} \approx 2.34 \AA$ (shown in Fig. 2(c) by the arrow) documents that at high temperatures the crystal structure of CeRuSn deviates distinctly from the more symmetric CeCoAl type.
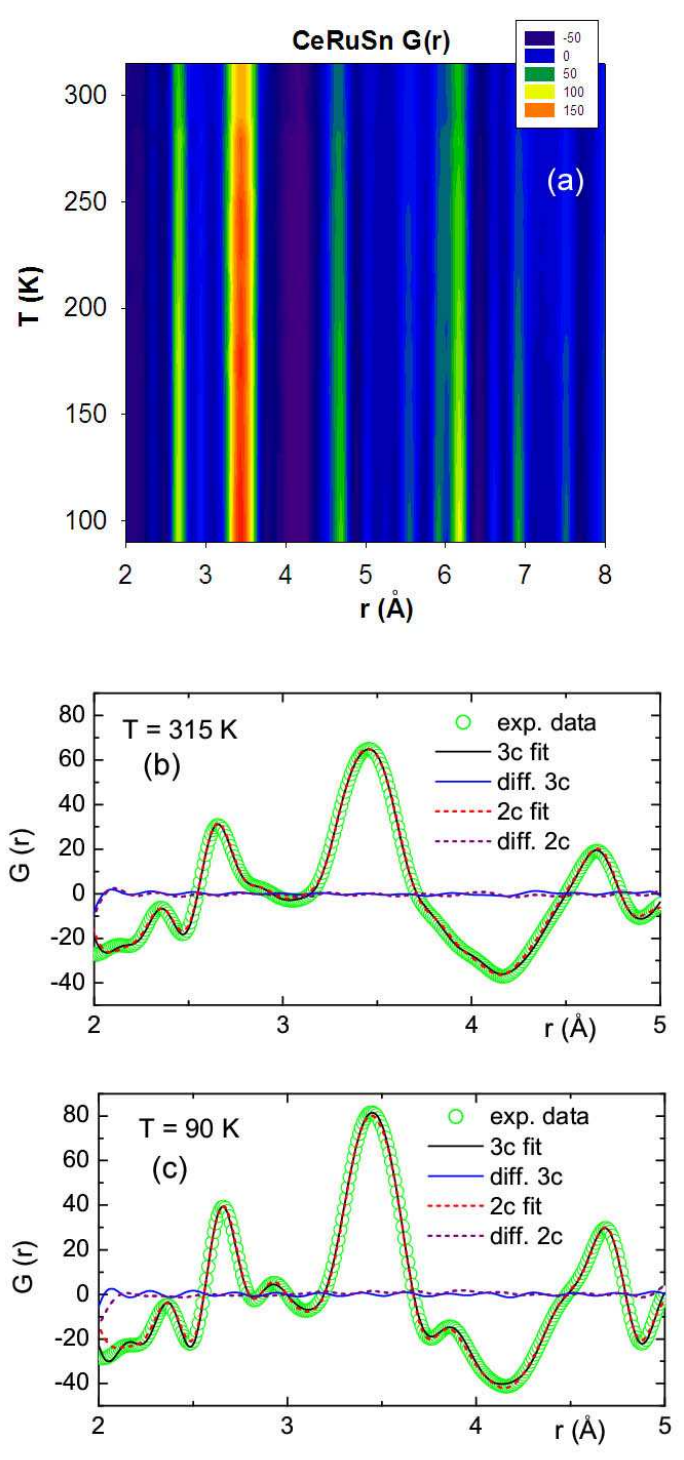

FIG. 3: (Color online) The temperature development of a portion of the CeRuSn PDF obtained upon cooling through the structural phase transition. The best fit to the $315 \mathrm{~K}$ data using the 2c (b) and 3c (c) structures as described in the text. The fitted range for these plots was 2 to $5 \AA$.

In Fig. 3(a) we show the thermal evolution of a portion of the CeRuSn experimental PDF obtained upon cooling between 315 and $90 \mathrm{~K}$, the lowest temperature in our experiment. Although CeRuSn exhibits in this temperature range a hysteretic structural transformation, the PDF does not change significantly. The observed PDF maxima move slightly towards lower $r$ spacing and sharpen up. The shift is more pronounced for larger $r$ values. This is characteristic of a lattice shrinkage and reduced displacement factors at lower temperatures. However, a closer inspection of the temperature dependencies of the fitted parameters reveal that the structural transforma- 


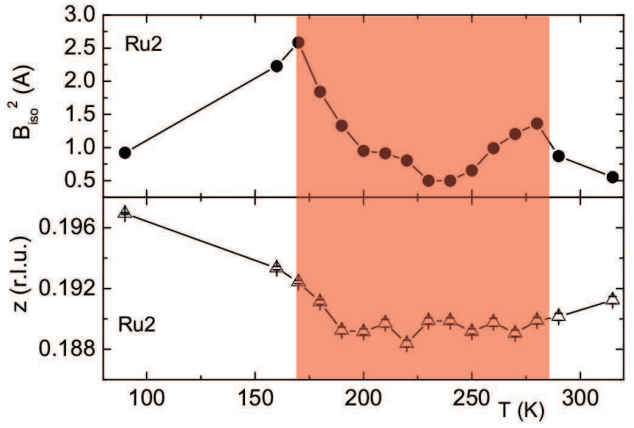

FIG. 4: (Color online) The temperature dependence of the isotropic displacement parameter and the positional parameter $\mathrm{z}$ of the $\mathrm{Ru} 2$ atom determined from the best fits to the $2 \mathrm{c}$ structural model between 2 and $5 \AA$. The hysteretic temperature range, in which the structural transition occurs upon cooling, is shaded.

tion manifests clearly in some atomic displacement and positional parameters.

The best fits, in which we treated symmetry-related atoms constrained to have equal isotropic displacement factors to the CeRuSn experimental PDF obtained at $315 \mathrm{~K}$ using the $2 \mathrm{c}$ and $3 \mathrm{c}$ structural models between 2 and $5 \AA$ are shown in Fig. 3(b). Surprisingly, there is hardly any difference in the fit quality suggesting that on a local scale both models describe the data equally well. This holds also for fits performed at $90 \mathrm{~K}$ shown in panel (c). Let us note that we have not experienced any radiation-related changes of our PDF data similar to history-related effects observed by Feyerherm et al. ${ }^{10}$. We have performed two data collection experiments, one with sample cooled down in the presence of the beam and the other on a sample that was exposed to high temperatures without X-ray beam on and cooled afterwards in the absence of radiation. The two resulting data sets were identical. It should be noted that in the present experiment the photoelectric absorption is by a factor of at least hundred smaller with respect to soft X-ray study ${ }^{10}$.

So, it seems that the fitting procedure does not bring anything useful regarding the crystal structure transformation. However, some isotropic displacement factors are found to have non-trivial temperature dependence and to be anomalously large even at low temperature. In Fig. 4 the temperature evolution of two of them - the isotropic displacement factor and the $\mathrm{z}$ positional parameter of the Ru2 atom obtained from the fits to the $2 \mathrm{c}$ structural model between 2 and $5 \AA$ are shown. The hysteretic region is clearly visible.

Anomalous isotropic displacement factor values are found mainly for atoms involved in the nearest $\mathrm{Ce}-\mathrm{Ru}$ bonding and there are basically two possibilities to explain them. They either reflect $(i)$ large thermal vibrations of atoms around their equilibrium positions or $(i i)$ reflect a distribution of more static atomic
TABLE I: Structural data for CeRuSn as determined from the pair distribution function at $315 \mathrm{~K}$ and $90 \mathrm{~K}$ on a fine powder. Both structures have the monoclinic $C 2 / m$ space group type, in which all the atoms occupy the $4 i(x, 0, z)$ Wyckoff position, however, with different $\mathrm{x}$ and $\mathrm{z}$ positional parameters. $\mathrm{B}$ denote the atomic displacement factors. The standard deviations in parentheses denote in the case of the atomic positions merely mathematical uncertainties resulting from fitting procedures.

\begin{tabular}{lcccc}
\hline \hline CeRuSn $\mathrm{T}=315 \mathrm{~K}$ & \multicolumn{3}{c}{$\mathrm{T}=90 \mathrm{~K}$} \\
model & $2 \mathrm{c}$ & $3 \mathrm{c}$ & \\
$\mathrm{a}(\AA)$ & $11.476(1)$ & & $11.4477(8)$ & \\
$\mathrm{b}(\AA)$ & $4.7122(5)$ & & $4.7117(4)$ & \\
c $(\AA)$ & $10.154(1)$ & & $15.1759(9)$ & \\
$\beta(\mathrm{deg})$ & $102.90(1)$ & & $103.373(8)$ & \\
$\chi^{2}$ & 4.13 & & 11.9 & \\
\hline Atom & $\mathrm{x}$ & $\mathrm{z}$ & $\mathrm{x}$ & $\mathrm{z}$ \\
Ce1 & $0.1369(1)$ & $0.4115(2)$ & $0.1292(2)$ & $0.1051(1)$ \\
Ce2 & $0.1255(2)$ & $0.9089(2)$ & $0.1242(1)$ & $0.4383(1)$ \\
Ce3 & - & - & $0.1458(1)$ & $0.7788(1)$ \\
Ru1 & $0.1810(3)$ & $0.6468(2)$ & $0.1762(1)$ & $0.2648(1)$ \\
Ru2 & $0.2051(2)$ & $0.1925(2)$ & $0.2014(2)$ & $0.6313(1)$ \\
Ru3 & - & - & $0.1978(2)$ & $0.9324(1)$ \\
Sn1 & $0.4289(2)$ & $0.3497(2)$ & $0.4155(2)$ & $0.0650(1)$ \\
Sn2 & $0.4003(2)$ & $0.8490(2)$ & $0.4084(2)$ & $0.3981(1)$ \\
Sn3 & - & - & $0.4255(2)$ & $0.7312(1)$ \\
\hline Atom & $B\left(A^{2}\right)$ & & $B\left(A^{2}\right)$ & \\
Ce1 & $0.86(3)$ & & $0.53(2)$ & \\
Ce2 & $0.93(3)$ & & $0.33(2)$ & \\
Ce3 & - & & $0.27(2)$ & \\
Ru1 & $1.07(5)$ & & $0.16(2)$ & \\
Ru2 & $1.01(4)$ & & $0.44(2)$ & \\
Ru3 & - & & $0.75(4)$ & \\
Sn1 & $0.51(2)$ & & $0.53(2)$ & \\
Sn2 & $0.80(2)$ & & $0.12(1)$ & \\
Sn3 & - & & $0.65(3)$ & \\
\hline \hline & & & & \\
\hline
\end{tabular}

positions that lead to a multitude of individual PDF peaks that overlap. Although the CeRuSn local order can be described by either of the two models, we argue that the non-trivial temperature dependence of parameters shown in Fig. 4 suggests that the latter model $(i i)$ is the right one. In this model, the local arrangement varies from place to place, leading to a distribution of interatomic distances and broadening of PDF peaks. On the long-range, a mean structure that results in superstructure Bragg reflections seen in $\mathrm{x}$ ray and neutron diffraction experiments ${ }^{3,5}$ is established.

The two possible models would have also different relations to the macroscopic bulk physical properties. For instance, the first (anomalously vibrating atoms) model (i) would imply a substantial contribution to the specific heat in the hysteretic critical region that would disappear again at low temperatures. Due to a continuous changes of the fitted parameters (especially of the displacement factors) one expects in the critical region a continuous 

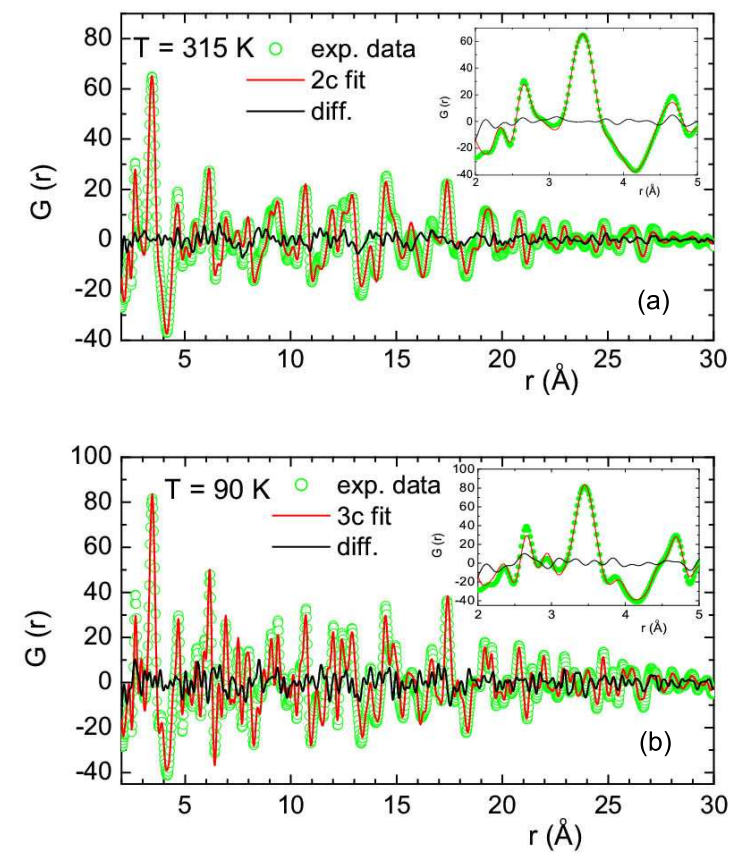

FIG. 5: (Color online) The best fits to the $315 \mathrm{~K}$ PDF data using the CeRuSn 2c structural model (a) and to the data taken at $90 \mathrm{~K}$ using the $3 \mathrm{c}$ structure model (b). Fits were performed in the range 2 to $30 \AA$.

appearance and disappearance of this additional specific heat contribution. Similar effects would be seen also in the temperature dependence of the electrical resisitivity and other physical properties. On the other hand, the positional-distribution model (ii) that represents in principle an order-order type transition would lead to anomalies at the onset and at the end of the hysteretic region, possible with plateaus between them.

The specific heat has been to date measured at high temperatures only on the polycrystalline sample and exhibits a hysteretic behavior that nicely matches the critical region from our data with indication of a first-order tansition $^{7}$. Further, while the electrical resistivity measured on a single crystal increases in a step-like manner for all three principal when entering the critical region on cooling followed by a decrease when leaving the hyteretic range $^{5}$, the electrical resistivity on a polycrystal exhibits only the latter transition that has the opossite $\operatorname{sign}^{7}$. The magnetic susceptibility looks differently for singe- and poly-crystalline samples as well. In the former case it shows two anomalies for both, decreasing and increasing temperature, the polycrystalline data reveal only the transition upon cooling. In both cases, however, steplike changes associated with a decrease of the density of states within the critical region are observed. These observations are corrobrated by hysteretic beavior of the CeRuSn thermopoower with a different slope above and below the critical region. All the bulk properties thus points to modifications in the electronic structure and/or creation of possibly anisotropic energy gaps at the Fermi surface. These are usually associated with crystal structure modifications (ii) rather than with smooth developments atomic vibrations $(i)$ existing in a limited range of temperatures. Perhaps the strongest evidence for the correctness of the distribution model comes from measurements of the relative length changes performed on a single-crystalline sample that showed a two-step monotonic change for all principal directions leading to a substantial reduction of the volume upon cooling ${ }^{5}$. This observation disagrees with the anomalously enhanced displacement which normally would lead to lattice expansion in the critical region.

In order to reveal the structural parameters of the mean CeRuSn crystal structure at high and low temperatures we have performed fits to the PDF data by extending the fit range to values that are substantially larger than the c lattice parameter. In Fig. 5(a) we show the best fit obtained to data taken at $315 \mathrm{~K}$ in a range from 2 to $30 \AA$ using the $2 \mathrm{c}$ structural model. Numerical results that are listed in Table I agree well with the literature data ${ }^{2,3,5}$. In particular, we observe within error bars the same anomalously large displacement factors (except for Ce2 and Ru1) as in the case of the neutron powder study. In the current work, atoms at different sites were treated separately but were allowed to have only isotropic displacement factors. The overall quality of the fit is rather good at the expense of a worse agreement at short distances (see the inset of Fig. 5(a) and compare with Fig. 3(b)). It should be noted that the fit to the $315 \mathrm{~K}$ data using the $3 \mathrm{c}$ structure is not better even if it contains more free parameters.

The best fit to data taken at $90 \mathrm{~K}$ with a $3 \mathrm{c}$ structure model is shown in Fig. 5(b). Numerical results are again listed in Table I. Also here one gets a reasonable but not perfect agreement with the experimental PDF data on a long range by worsening the good agreement on a local scale (see the inset of Fig. 5(b) and compare with Fig. 3(c)). The fit to $90 \mathrm{~K}$ data using the 3c model is clearly preferred over the $2 \mathrm{c}$ model. The $\chi^{2}$ for the latter fit is by a factor of two larger with respect to the former one. This suggests that the necessity to increase the number of free fit parameters in order to explain the observed PDF data is essential. On the other hand, the agreement is far from being ideal. We argue at this point that the crystal structure of CeRuSn is even more complicated and that the $3 \mathrm{c}$ model is only an approximation to the reality.

A possibility to increase the agreement between the structure model and PDF data would be a mixture of different crystallographic phases. In fact, earlier soft X-ray synchrotron work identified a presence of several competing phases having few different periodicities ${ }^{10}$. These can be considered as being built from identical structural blocks (in the work of Feyerherm et al. denoted as A and B, depending on whether they contain a $\mathrm{Ce} 1$ or $\mathrm{Ce} 2$ site) that are stacked along the $c$-axis in various ways, possibly containing stacking faults. Let us note that such a 
model is in full agreement with our data as it leads to a wider spread of interatomic distances as compared to pure $2 \mathrm{c}$ or $3 \mathrm{c}$ structures. Also our powder diffraction data were interpreted in terms of phase coexistence ${ }^{3}$. Neither of the methods has been able, however, to solve the problem completely. The synchrotron experiment could concentrate only on a small portion of the reciprocal space without a possibility to refine crystallographic details and the powder data have suffered from overlapping and/or small intensity Bragg reflections involved. A mixture of different related crystallographic phases (or, alternativelly, differently stacked basic building blocks) would naturally explain a better agreement between the best fit and PDF data within a short fit range and worse agreement in a wide range. However, the number of free parameters is then unreasonably high, hampering reliable interpretation of the results.

Another possibility to explain the differences between the fit and the data would be a subtle structural distortion upon lowering the temperature leading to further lowering of the symmetry from the monoclinic to a triclinic lattice and yet another one would be a more complicated crystal structure such a modulated one found in some lanthanides on a long scale ${ }^{19}$ or in magnetite on a local scale ${ }^{20}$.

\section{CONCLUSIONS}

The pair distribution functions collected at 90 and $315 \mathrm{~K}$ on a fine CeRuSn powder that consists from broadened peaks suggests that the local symmetry in this compound does not modify upon cooling significantly. However, anomalous atomic displacement factor values suggest that a relatively good agreement between the best fits to $2 \mathrm{c}$ or $3 \mathrm{c}$ models with PDF spectra could be misleading. Although a distribution of atomic positions or strong atomic vibrations would equally well explain the broadening of PDF signal, the temperature dependence of fitted parameters strongly suggest that a distribution of interatomic distances for particular atomic pairs exist within the structure. A natural explanation for the distribution would be a coexistence of different competing crystallographic phases. A re-population of different phases or a re-arrangement of atomic positions upon cooling then leads to the observed macroscopic modifications of the crystal structure. At low temperatures the crystal structure model having a three times larger c lattice parameter with respect to the $\mathrm{CeCoAl}$ type is clearly preferred. However, a limited agreement with the PDF data suggests that the real crystal structure of CeRuSn at low temperatures is even more complicated.

\section{Acknowledgments}

We acknowledge greatly the ESRF for the allocated beamtime.
* Electronic address: prokes@helmholtz-berlin.de

1 P. M. Chaikin and T. C. Lubensky Principles of Condensed Matter (Cambridge University Press, Cambridge 1995) 1.

2 J. F. Riecken, W. Hermes, B. Chevalier, R.-D. Hoffmann, F. M. Schappacher, and R. Pöttgen, Z. Anorg. Allg. Chem. 633, (2007) 1094.

${ }^{3}$ K. Prokeš, J. A. Mydosh, O. Prokhnenko, W.-D. Stein, S. Landsgesell, W. Hermes, R. Feyerherm, and R. Pöttgen, Phys. Rev. B 87, (2013) 094421.

${ }^{4}$ S. F. Matar, J. F. Riecken, B. Chevalier, R. Pöttgen, A. F. Al Alam, and V. Eyert, Phys. Rev. B 76, (2007) 174434.

5 J. Fikáček, J. Prokleška, M. Míšek, J. Custers, S. Daniš, J. Prchal, V. Sechovský and I. Císařová, Phys. Rev. B 86, (2012) 054108.

6 J. Fikáček, J. Prokleška, J. Prchal, J. Custers, and V. Sechovský and I. Císařová, J. Phys.: Condens. Matter 25 (2013) 416006.

7 J. A. Mydosh, A. M. Strydom, M. Baenitz, B. Chevalier, W. Hermes, and R. Pöttgen, Phys. Rev. B 83, (2011) 054411.

8 J. W. Allen and L. Z. Liu, Phys. Rev. B 46, (1992) 5047.

9 M. J. Lipp, D. Jackson, H. Cynn, C. Aracne, W. J. Evans, and A. K. McMahan, Phys. Rev. Lett. 101, (2008) 165703.

10 R. Feyerherm, E. Dudzik, S. Valencia, J. A. Mydosh, Y.K. Huang, W. Hermes, and R. Pöttgen, Phys. Rev. B 85,
(2012) 085120.

11 H. J. Kim, C. D. Malliakas, A. T. Tomic, S. H. Tessmer, M. G. Kanatzidis, and S. J. L. Billinge, Phys. Rev. Lett. 96, (2006) 226401.

12 E. S. Božin, A. S. Masadeh, Y. S. Hor, J. F. Mitchell, and S. J. L. Billinge, Phys. Rev. Lett. 106, (2011) 045501.

13 S. J. L. Billinge, J. Solid State Chem. 181, (2008) 1698.

14 Y.-I. Kim, K. Page, A. M. Limarga, D. R. Clarke, and R. Seshadri, Phys. Rev. B 76, (2007) 115204.

15 D. P. Shoemaker, L. J. Li, and R. Seshadri, J. Am. Chem. Soc., 131 (2009), 11450.

16 A. P. Hammersley, S. O. Svensson, A. Thompson, H. Graafsma, A. Kvick, and J-P. Moy, Rev. Sci. Instr., 66, 2729 (1995).

17 S. A. J. Kimber, computer code iPDF based on Igor Pro, ESRF unpublished (2011)

18 C. L. Farrow, P. Juhas, J. W. Liu, D. Bryndin, E. S. Božin, J. Bloch, Th. Proffen and S. J. L. Billinge, J. Phys.: Condens. Matter 19 (2007) 335219.

19 R. J. Husband, I. Loa, G. W. Stinton, S. R. Evans, G. J. Ackland and M. I. McMahon, Phys. Rev. Lett. 109, (2012) 095503.

20 M. S. Senn, J. P. Wright and J. P. Attfield, Nature 481, (2012) 173. 